\title{
Les troubles cognitifs, comportementaux et d'apprentissage chez les enfants nés prématurément : une revue de littérature
}

Los problemas cognitivos, comportamentales y de aprendizaje en niños prematuros: una revisión de la literatura

Prejuízos cognitivos, comportamentais e de aprendizagem em crianças prematuras: uma revisão

Cognitive, behavioral and learning problems in premature children: a review

\section{Mélissa Sue Sayeur ${ }^{1,2,3}$, Michelle McKerral ${ }^{2,3} \&$ Maryse Lassonde ${ }^{1,2,3}$}

\author{
${ }^{1}$ Centre Hospitalier Universitaire Sainte-Justine, Montréal, Canada. ${ }^{2}$ Centre de Recherche de Neuropsychologie et Cognition \\ (CERNEC), Canada. ${ }^{3}$ Université de Montréal, Canada.
}

\begin{abstract}
Résumé
Les naissances prématurées $(\leq 37$ semaines gestationnelles) ont été identifiées comme l'une des causes de mortalité périnatale les plus importantes dans les sociétés industrialisées. Grâce à l'amélioration des soins pré- péri et post-nataux, il est maintenant possible de sauver ces enfants à des âges et des poids gestationnels significativement plus bas qu'il était possible de le faire auparavant. Avec l'augmentation du taux de survie, de plus en plus d'études s'intéressent aux impacts de la prématurité sur le devenir neurodéveloppemental des enfants nés à 37 semaines gestationnelles et moins. En effet, les études réalisées à ce jour indiquent que les enfants nés prématurément sont à haut risque d'éprouver des problèmes d'ordre cognitif, comportemental et d'apprentissage, et que ceux-ci peuvent se maintenir jusqu'à l'âge scolaire, voire jusqu'à l'adolescence et/ou à l'âge adulte. Cette revue de littérature a pour but de recenser les divers troubles cognitifs, comportementaux et d'apprentissage documentés dans les récentes études s'intéressant aux conséquences de la prématurité. Mots clés : Prématurité, cognition, comportement, troubles d'apprentissage.
\end{abstract}

\section{Resumen}

Los nacimientos prematuros ( $\leq 37$ semanas de gestación) se consideran una de las causas más importantes de mortalidad perinatal en las sociedades industrializadas. Hoy en día, gracias a la mejora de los cuidados pre- peri- y post-natales, es posible salvar a niños con edades y pesos gestacionales significativamente menores que anteriormente. Con el aumento de la tasa de supervivencia, cada vez más estudios se interesan por el impacto de la prematuridad en el neurodesarrollo de niños nacidos a las 37 semanas de gestación o incluso antes. En efecto, los estudios realizados hasta este momento indican que los niños prematuros tienen alto riesgo de presentar problemas de orden cognitivo, comportamental y de aprendizaje. Los mismos pueden mantenerse hasta la edad escolar e incluso hasta la adolescencia y la edad adulta. Esta revisión de la literatura tiene por objeto presentar los distintos problemas cognitivos, comportamentales y de aprendizaje documentados en estudios recientes interesados por las consecuencias de la prematuridad.

Palabras clave: Prematuridad; cognición; comportamiento; problemas de aprendizaje.

\section{Resumo}

Nascimentos pré-termo ( $\leq 37$ gestational weeks) tem sido identificados como uma das causas mais importantes de mortalidade perinatal em sociedades industrializadas. Com a melhoria dos serviços pré- peri- e pós-natais, atualmente é possível salvar estas crianças com significativos baixos peso e idade gestacional. Frente às crescentes taxas de sobrevivência, cada vez mais estudos abordam o impacto da prematuridade no neurodesenvolvimento de crianças nascidas até 37 semanas de gestação. Pesquisas até então sugerem que crianças prematuras apresentam alto risco de dificuldades cognitivas, comportamentais e de aprendizagem, sendo que estas últimas podem se prolongar até a idade escolar, mantendo-se até mesmo na adolescência e/ou vida adulta. Esta revisão visou a identificar tais dificuldades em investigações recentes sobre as consequências da prematuridade.

Palavras-chave: Prematuridade; cognição; comportamento; dificuldades de aprendizagem.

Artícle reçu: 17/08/2011; Artícle revisé: 29/08/2011; Artícle accepté: 30/08/2011.

Mélissa Sue Sayeur, Centre de recherche, Centre Hospitalier Universitaire Sainte-Justine, Montréal, Québec, Canada ; Centre de Recherche de Neuropsychologie et Cognition (CERNEC), Montréal, Québec, Canada. Département de Psychologie, Université de Montréal, Québec, Canada. Michelle McKerral, Centre de Recherche de Neuropsychologie et Cognition (CERNEC), Montréal, Québec, Canada. Département de Psychologie, Université de Montréal, Québec, Canada. Maryse Lassonde, Centre de recherche, Centre Hospitalier Universitaire Sainte-Justine, Montréal, Québec, Canada ; Centre de Recherche de Neuropsychologie et Cognition (CERNEC), Montréal, Québec, Canada ; Département de Psychologie, Université de Montréal, Québec, Canada.

Adresse de correspondance: Professeure Maryse Lassonde, Département de Psychologie, Université de Montréal, C.P. 6128, Succ. Centre-Ville, Montréal, Québec, Canada, H3C 3J7 ; fax : 1-514-343-5787.

E-mail: Maryse.lassonde@ umontreal.ca

DOI: $10.5579 / \mathrm{rnl} .2011 .0071$ 


\section{Abstract}

Preterm births ( $\leq 37$ gestational weeks) have been identified as one of the most important cause of perinatal mortality in industrialized societies. With improvement in pre- peri- and post-natal care, it is now possible to save these children at significantly lower weight and gestational age than previously. With increasing survival rates, more and more studies address the impact of prematurity on the neurodevelopmental outcomes of children born at or before 37 weeks of gestation. Studies to date indicate that children born prematurely are at high risk of experiencing cognitive, behavioural and learning problems, and that the latter can last up to school age and even into adolescence and/or adulthood. The aim of this review is to identify the various cognitive, behavioural and learning difficulties documented in recent studies investigating the consequences of prematurity.

Key words: Prematurity; cognition; behaviour; learning problems.

Les naissances prématurées ont été identifiées comme l'un des problèmes de santé périnatale les plus importants dans les sociétés industrialisées et causent 75 à $85 \%$ de toutes les mortalités périnatales au Canada (Agence de la Santé Publique du Canada, 2004). En octobre 2006, les limites de la viabilité ont été repoussées à 21 semaines de gestation, où le plus jeune bébé prématuré survivant a été déclaré (New Scientist \& Reuters, 2007). À l'échelle mondiale, environ 13 millions de bébés naissent avant d'atteindre la $37^{\mathrm{e}}$ semaine de gestation et il est estimé qu'un million d'entre eux meurt chaque année (Organisation Mondiale de la Santé, 2010). Au Canada, le taux de naissance prématurée se situe approximativement à $7.1 \%$, alors qu'aux États-Unis ce taux est estimé à $12.8 \%$, ce qui représente une augmentation de 36\% depuis le début des années 1980 (Agence de la Santé Publique du Canada, 2004). Non seulement le nombre de naissances prématurées augmente avec les années, mais l'amélioration des soins pré- péri- et post-nataux accroît le taux de survie de ces enfants à des âges et poids gestationnels significativement plus bas qu'il était possible quelques années auparavant (Allen, 2002).

Toutefois, malgré une augmentation du taux de survie, l'incidence des problèmes neurodéveloppementaux chez les enfants nés prématurément demeure élevée. En effet, les lésions cérébrales sont une cause majeure de troubles neurologiques subséquents chez ceux-ci (Nolin \& Laurent, 2004). La littérature indique à ce sujet que l'incidence de conditions telles que la ventriculomégalie, les paralysies et les hémorragies cérébrales, les restrictions de croissance intrautérine, ainsi que les hypoxies-ischémies, est plus élevée chez les enfants prématurés en comparaison aux enfants nés à terme (du Plessis \& Volpe, 2002; Ment et al., 1999; Scherjon, Briet, Oosting, \& Kok, 2000; Volpe, 2001). Par ailleurs, ces études indiquent que les lésions cérébrales associées à la prématurité s'avèrent aussi associées à l'apparition de divers déficits fonctionnels une fois l'enfant parvenu à l'âge scolaire. Toutefois, même en l'absence de lésion cérébrale à la naissance, les enfants prématurés peuvent présenter des déficits cognitifs et des difficultés d'apprentissage scolaire (Saigal \& Doyle, 2008). Étant donné que le développement du système nerveux est affecté par la prématurité, ces enfants sont également plus à risque d'éprouver des déficits sensoriels dont la gravité augmente à mesure que l'âge gestationnel et le poids à la naissance diminuent (Nolin \& Laurent, 2004). Conséquemment, l'incidence élevée des séquelles neurologiques et sensorielles a un impact central sur le développement des habiletés cognitives chez l'enfant prématuré. Heureusement, plusieurs d'entres eux connaîtront un développement normal aux plans intellectuel et cognitif, alors qu'il en sera autrement pour d'autres, qui devront faire face à divers déficits affectant ces fonctions.
Dans ce contexte, nous révisons de récentes études s'étant intéressées au développement des fonctions cognitives, comportementales et affectives, ainsi que le rendement académique chez les enfants nés prématurément, afin de dresser un portrait général des impacts à moyen et long terme sur leur fonctionnement.

\section{La prématurité et le petit poids à la naissance}

\section{Définition}

Un enfant est considéré comme prématuré s'il naît avant la $37^{\mathrm{e}}$ semaine de gestation à partir de la dernière date des menstruations et qu'il a un poids inférieur à $2500 \mathrm{~g}$ (Organisation Mondiale de la Santé Santé, 2010). Il existe différents stades de prématurité selon le nombre de semaines gestationnelles que possède l'enfant à sa naissance. Il est question de petite prématurité s'il naît entre la $33^{\mathrm{e}}$ et la $36^{\mathrm{e}}$ semaine de gestation, de grande prématurité s'il naît entre les $28^{\mathrm{e}}$ et $32^{\mathrm{e}}$ semaines, de très grande prématurité s'il naît entre la $26^{\mathrm{e}}$ et la $27^{\mathrm{e}}$ semaine et d'extrême prématurité lorsque l'enfant naît avant d'atteindre la $26^{\mathrm{e}}$ semaine de gestation.

La survie des bébés prématurés est intimement liée à la durée de leur gestation et à leur poids à la naissance, deux facteurs qui sont fréquemment utilisés pour décrire la prématurité chez ces derniers. Cependant, le poids à la naissance serait une mesure plus fiable que l'âge gestationnel et serait donc plus souvent employé comme marqueur de la prématurité (Taylor, Minich, Bangert, Filipek et Hack, 2004). À la naissance, le bébé doit peser moins de $2500 \mathrm{~g}$ pour être considéré comme un bébé à faible poids, moins de $1500 \mathrm{~g}$ pour un bébé à très faible poids et moins de $1000 \mathrm{~g}$ pour un bébé à poids extrêmement faible (Aylward, 2002). Même si ces derniers constituent seulement $1 \%$ de toutes les naissances, ils contribuent en grande partie au taux de mortalité néonatale (Taylor, Klein et Hack, 2000). En effet, il est rapporté que seulement $54 \%$ des bébés qui naissent avec un poids entre 501 et $750 \mathrm{~g}$ survivent, alors que ce taux passe à $97 \%$ chez les bébés naissant avec un poids entre 1250-1500g (Zelkowitz, 2004).

Troubles cognitifs, comportementaux et d'apprentissage
associés

Prévalence

Au fil des années, de plus en plus d'études se sont intéressées aux conséquences à moyen et à long-terme de la prématurité et du petits poids à la naissance. Comme le taux de survie de ces bébés augmente, l'incidence des déficits cognitifs, intellectuels, des troubles d'apprentissage, des troubles du déficit d'attention avec hyperactivité (TDAH), des problèmes comportementaux et sociaux augmente également. 


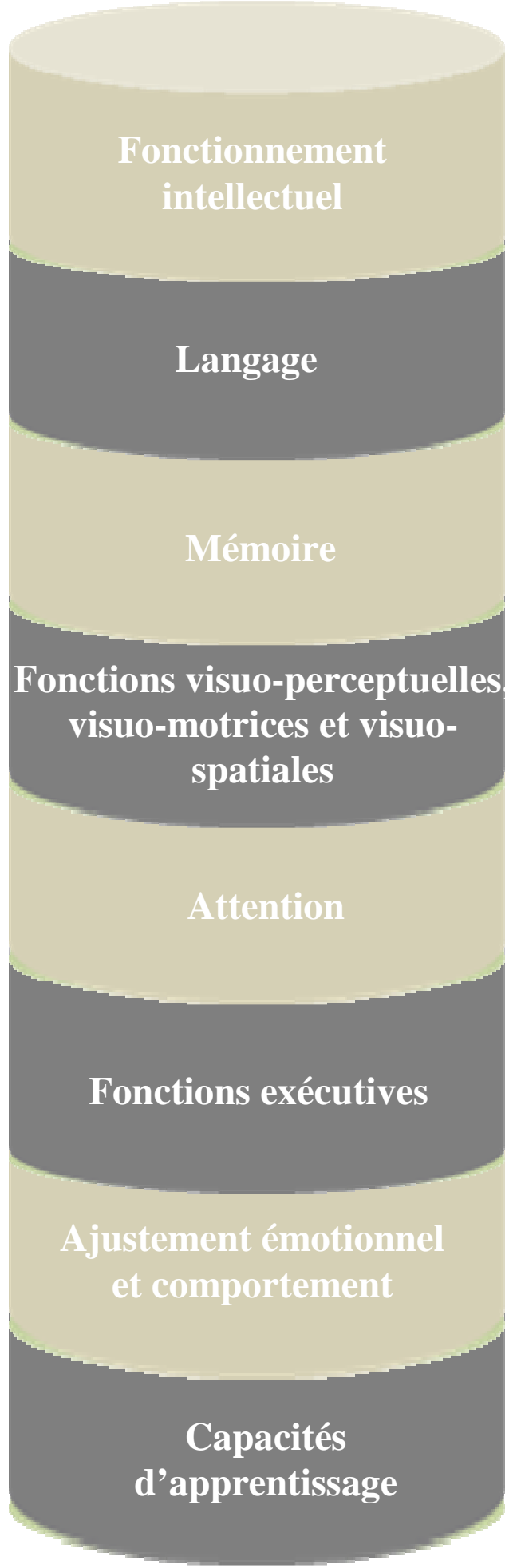

(Anderson \& Doyle, 2008; Anderson, Doyle, \& Victorian Infant Collaborative Study, 2004; Aylward, 2002; Bayless, Pit-ten Cate, \& Stevenson, 2008; Bhutta, Cleves, Casey, Cradock, \& Anand, 2002; Hack \& Taylor, 2000; Méio et al., 2004; Moster, Lie, \& Markestad, 2008; Raz, Debastos, Newman, \& Batton, 2009)

(Briscoe, Gathercole, \& Marlow, 1998; Guarini et al., 2009; Jansson-Verkasalo et al., 2004; Le Normand \& Cohen, 1999; Taylor, Hack et Klein, 1998; Taylor, Klein, Minich et Hack, 2000; Valleur, Magny, Rigourd, \& Kieffer, 2004; Wolke, Samara, Bracewell, Marlow, \& Group, 2008)

(Caravale, Tozzi, Albino, \& Vicari, 2005; Dewey, Crawford, Creighton, \& Sauve, 1999; Taylor, Klein, Minich et Hack, 2004; Taylor, Minich, Bangert, Filipek et Hack, 2000; Taylor et al. 2000; Vicari, Caravale, Carlesimo, Casadei, \& Allemand, 2004)

(Aylward, 2002; Caravale, et al., 2005; Davis, Burns, Wilkerson, \& Steichen, 2005; Dewey, et al., 1999; Foulder-Hughes \& Cooke, 2003; Goyen, Lui, \& Woods, 1998; Isaacs, Edmonds, Chong, Lucas, \& Gadian, 2003; Jongmans et al., 1996; O’Reilly et al., 2010; Saigal, Stoskopf, Streiner et Burrows, 2001)

(Aylward, 2002; Caravale, et al., 2005; Elgen, Lundervold, \& Sommerfelt, 2004; Hack et al., 2009; Marlow, Hennessy, Bracewell, Wolke, \& Group, 2007; Taylor et al. 1998; Taylor, Klein, et Hack, 2000; Taylor et al. 2000)

(Anderson, et al., 2004; Bayless \& Stevenson, 2007; Mahone \& Hoffman, 2007; Marlow, et al., 2007; Taylor et al., 2000)

(Anderson \& Doyle, 2008; Baron, Ahronovich, Erickson, Gidley Larson, \& Litman, 2009; Bayless, et al., 2008; Botting, Powls, Cooke, \& Marlow, 1997; Grunau, Whitfield, \& Fay, 2004; Hack et al., 1994; Hack, et al., 2009; Horwood, Mogridge, \& Darlow, 1998; Indredavik et al., 2004; Moster, et al., 2008; Stephens \& Vohr, 2009; Taylor, Klein et Hack, 2000; Taylor et al. 2000; Valleur, et al., 2004; Weisglas-Kuperus, Koot, Baerts, Fetter, \& Sauer, 1993)

Figure 1. Classement des études recensées s'intéressant au fonctionnement cognitif, comportemental et aux capacités d'apprentissage chez les enfants prématurés 
Contrairement aux problèmes moteurs et sensoriels qui peuvent être identifiés durant la première année de vie des enfants prématurés, les problèmes d'ordre cognitif sont plutôt mis en évidence en début de scolarisation où leurs fonctions intellectuelles, cognitives et psychosociales sont grandement sollicitées (Anderson \& Doyle, 2008). Avant cet âge, il est plutôt question de «retard» ou de «délai » dans le développement cognitif que de déficits cognitifs spécifiques. À cet effet, des études rapportent qu'à l'âge de 30 mois, 30\% à $40 \%$ des bébés nés à un âge gestationnel de 26 semaines et moins montrent un délai modéré à sévère au niveau cognitif ou moteur (Doyle \& the Victorian Infant Collaborative Study Group, 2004; Jacobs, O'Brien, Inwood, Kelly, \& Whyte, 2000; Raz, et al., 2009). Non seulement ces retards persistent jusqu'à l'âge de six ans, mais à cet âge leur prévalence atteint les 46\% (Marlow, Wolke, Bracewell, Samara, \& E. PICure Study Group, 2005). Avec des taux aussi élevés, il n'est pas étonnant que les enfants prématurés et de petits poids soient moins performants à l'école que leur pairs nés à terme, et qu'ils requièrent plus de soutien de la part des ressources spécialisées (ex. orthopédagogue, orthophoniste, ergothérapie, etc.) au cours de leur cheminement (Valleur, et al., 2004).

En plus d'exercer une influence néfaste sur le rendement académique de l'enfant, les déficits cognitifs à long-terme peuvent s'accompagner d'une diminution de l'estime de soi, de l'intégration sociale et du fonctionnement global (Anderson \& Doyle, 2008; Aylward, 2002). Les difficultés scolaires semblent persister jusqu'à l'adolescence chez les enfants nés prématurément ou considérés comme ayant un petit poids à la naissance, où $72 \%$ de ceux nés avec un poids de $750 \mathrm{~g}$ et moins exhibent ce type de problèmes (Saigal \& Doyle, 2008). Ce taux diminue à 53\% pour les enfants dont le poids varie entre 750 et $1000 \mathrm{~g}$, mais demeure très supérieur en comparaison aux enfants nés à terme dont le taux de difficultés scolaires se situe à $12 \%$. En d'autres termes, la littérature suggère un effet de gradient en ce qui concerne le fonctionnement cognitif des enfants prématurés; un peu plus de la moitié d'entre eux (52\%) ne présentent aucune difficulté à l'âge de quatre ans alors qu'à l'âge de huit ans, ce taux passe à $31 \%$ (Saigal, Szatmari, Rosenbaum, Campbell, \& King, 1990; Szatmari, Saigal, Rosenbaum, Campbell, \& et al., 1990). Il est donc important de bien évaluer ces enfants tôt dans leur développement, mais également d'assurer un suivi puisqu'un fonctionnement normal à l'âge préscolaire n'assure pas un fonctionnement normal une fois sur les bancs d'école. La section qui suit exposera les différentes atteintes cognitives ainsi que les troubles émotionnels, comportementaux et d'apprentissage, pouvant être observés chez les enfants nés prématurément. La figure 1 permet d'apprécier les travaux recensés en fonction du domaine principalement étudié par les auteurs.

\section{Le fonctionnement intellectuel}

La mesure du quotient intellectuel (QI) procure généralement une base solide sur laquelle s'appuyer afin d'évaluer le fonctionnement cognitif global d'un individu. Chez les enfants prématurés, la moyenne du QI tend à s'abaisser avec la diminution de l'âge gestationnel (Bhutta, et al., 2002; Johnson, 2007). En effet, une étude rapporte que le QI moyen pour des enfants nés à 26 semaines de gestation et moins est inférieur de 0.8 à 1.5 écart-type comparativement au QI moyen d'enfants nés à terme, alors qu'il est inférieur de
0.3 à 0.5 écart-type chez les enfants nés entre 26 et 31 semaines gestationnelles (Anderson \& Doyle, 2008). D'autres études se sont intéressées au fonctionnement intellectuel d'un groupe d'enfants prématurés ( $<28$ semaines) lorsqu'ils furent âgés de huit ans et ont permis de mettre en évidence encore une fois un QI global inférieur à celui des enfants du groupe contrôle nés à terme (Anderson, et al., 2004). Cette divergence de profil intellectuel semble même se maintenir à l'âge adulte où, à l'âge de 20 ans, les prématurés montrent des scores de QI significativement plus faibles que leurs pairs nés à terme (Hack \& Taylor, 2000).

D'autres auteurs, qui se sont intéressés à la prévalence de déficience intellectuelle (i.e. QI < -2 écart-type par rapport à la moyenne) chez les enfants nés prématurément, ont montré que le taux de déficience varie entre 4.4 à $35 \%$ selon les études, comparativement à $0.4 \%$ chez les enfants nés à terme (Moster, et al., 2008; Roberts, Anderson, \& Doyle, 2009). De plus, les enfants de 4-5 ans nés entre 23 et 25 semaines gestationnelles présenteraient un QI de performance (QIP) significativement plus bas que leur QI verbal (QIV) comparativement à ceux nés entre 26 et 27 semaines, ce qui soutient les résultats d'autres études montrant que la prévalence des déficits intellectuels augmente avec une diminution de l'âge gestationnel (Aylward, 2002; Raz, et al., 2009). L'étude de Méio, Lopes, Morsch, Monteiro, Rocha, Borges et al. (2004) a démontré à des résultats similaires; les enfants prématurés ont obtenu des scores significativement plus faibles que les enfants nés à terme au WPPSI-R, et ce, tant pour le QI global, verbal, et non-verbal. En effet, les scores moyens se situaient entre 65 et 89 , ce qui montre que le rendement intellectuel des enfants prématurés se retrouve entre la basse moyenne et le seuil déficitaire. Selon l'étude de Bayless, Pit-Ten Cate et Stevenson (2008), un QI plus faible serait associé avec un bien-être émotionnel et des habiletés pro-sociales plus faibles chez les enfants prématurés, les rendant encore plus à risque d'éprouver des difficultés dans leur vie quotidienne, que ce soit à l'école, à la maison ou lorsqu'ils entrent en relation avec leur pairs.

Le langage

Les atteintes langagières sont souvent rapportées chez les enfants très prématurés $(<32$ semaines gestationnelles) et de très petits poids $(<1500 \mathrm{~g})$ (Briscoe, et al., 1998; Jansson-Verkasalo, et al., 2004; Wolke, et al., 2008). Elles peuvent se manifester par des difficultés d'acquisition du lexique phonologique et morphologique et des habiletés de dénomination. Par exemple, l'étude de Guarini, Sansavini, Fabbri, Alessandroni, Faldella et Karmiloff-Smith (2009) a mis en évidence des habiletés grammaticales, un vocabulaire et un lexique phonologique moins développés chez les enfants prématurés en comparaison aux enfants nés à terme (âge moyen 72.6 mois, E.T. =3.5), et ce, même en contrôlant pour le rendement intellectuel et les complications médicales liées à la prématurité. Par contre, une autre étude suggère que le vocabulaire et le langage réceptif des enfants prématurés sont relativement normaux alors que les processus verbaux plus complexes tels que la syntaxe, la production verbale seraient déficients chez ces enfants (Le Normand \& Cohen, 1999). D'autres auteurs croient plutôt que malgré une plus grande prévalence de difficultés langagières chez ces derniers, elles seraient de nature non-spécifique et associées à des difficultés cognitives plus générales (Wolke, 
et al., 2008). Quoi qu'il en soit, ces problèmes langagiers sont observables dès l'âge préscolaire chez les enfants pesant moins de $750 \mathrm{~g}$ à la naissance et persistent jusqu'à l'âge scolaire, voire au-delà de l'adolescence, pour certaines fonctions langagières de haut-niveau (i.e. identifier des synonymes, pragmatique, fluence verbale) (Taylor, Klein et Hack, 1998; Taylor, Klein, Minich et Hack, 2000).

À l'école, ces difficultés langagières se traduisent par des troubles de lecture, d'écriture, ainsi que des troubles d'apprentissage et seraient prédictifs de la dyslexie et de la dyscalculie (Jansson-Verkasalo, et al., 2004; Valleur, et al., 2004). En effet, la représentation des sons de la parole et leur analyse perceptuelle permettent le développement du lexique mental et de l'habileté de dénomination. Ces habiletés contribuent à leur tour, au développement de la capacité de lecture une fois à l'âge scolaire. Chez les enfants prématurés de petits poids, le traitement auditif et les habiletés de dénomination apparaissent comme étant déficitaires, ce qui contribuerait à leurs difficultés d'acquisition de langage et à l'apparition de troubles qui leur sont associés (i.e. dyslexie, trouble de dénomination, difficulté à différencier les phonèmes et les syllabes, etc.) (Jansson-Verkasalo, et al., 2004).

\section{Mémoire}

En lien avec les difficultés langagières, il semble que ces enfants performent moins bien aux tâches de mémoire verbale (ex. CVLT-C) comparativement à leur pairs nés à terme avec un encodage et un rappel significativement inférieurs, en plus de présenter plus d'intrusions lors du rappel des listes de mots (Taylor, et al., 2004; Taylor et al., 2000; Taylor, Klein, Minich et Hack, 2000). Encore une fois, ces déficits semblent se maintenir à l'adolescence. Il en va de même pour la mémoire visuelle qui est aussi rapportée comme étant déficitaire chez les enfants prématurés âgés de 3 et 4 ans (Caravale, et al., 2005; Taylor, et al., 2004; 2000), ainsi que ceux âgés de 7 à 9 ans (Taylor, et al., 2004). L'étude de Dewey, Crawford, Creighton et Sauve (1999), qui s'est intéressée au fonctionnement neuropsychologique chez des enfants prématurés de poids extrêmement faible $(<1500 \mathrm{~g}$, âge moyen $=9.1$, E.T. $=2.5)$, a mis en évidence des profils différents selon la présence ou non de déficit neurosensoriel chez l'enfant. Les auteurs ont identifiés deux sous-groupes d'enfants prématurés de poids extrêmement faible sur la base de leurs résultats au McCArty Scale of Children's Abilities ou au Stanford Binet Intelligence Scale Fourth Edition à l'âge de trois ans : ceux dont le développement était « suspect » (i.e. à risque de développer des problèmes d'apprentissage) et ceux dont le développement était normal. Pour être identifiés comme ayant un développement «suspect», les enfants devaient obtenir au moins 15 points d'écart (scores standards) entre deux domaines cognitifs à l'une ou l'autre des batteries d'intelligence administrée, ou avoir un faible ensemble de scores standards à l'une ou l'autre de ces batteries. Grâce à cette méthode, les auteurs ont pu démontrer que les enfants de poids extrêmement faible ayant été identifiés comme «suspect» au plan développemental à l'âge de trois ans présentaient des déficits au plan de leurs habiletés mnésiques verbales et visuelles une fois à l'âge scolaire, tel que mesuré par la batterie Wide Range Assessment of Memory and Learning (WRAML). À l'opposé, ces mêmes habiletés ne semblaient pas atteintes chez les enfants prématurés de poids extrêmement faible se développant normalement. Les résultats de cette étude montrent que les enfants identifiés comme étant sujet à développer des problèmes développementaux dus à des atteintes des fonctions cognitives à l'âge de trois ans, continuent de montrer des déficits mnésiques une fois à l'âge scolaire. Enfin, une étude suggère également des difficultés à maintenir l'information spatiale en tête et à la manipuler (i.e. mémoire de travail spatiale) chez les enfants prématurés âgés de 3-4 ans, tel que mesuré par le test Memory for location 2 (Vicari, et al., 2004). En outre, ces données semblent indiquer que les enfants prématurés sont moins efficaces que les enfants nés à terme en ce qui concerne l'apprentissage d'information verbale et visuelle et seraient donc, plus à risque d'oublier des détails importants en classe.

Fonctions visuo-perceptuelles, visuo-motrices et visuospatiales

Parmi les autres déficits neuropsychologiques chez les enfants prématurés, on rapporte notamment des déficits visuo-perceptuels, visuo-moteurs, visuo-spatiaux et ce, tant à l'âge préscolaire qu'à l'adolescence (Caravale, et al., 2005; Foulder-Hughes \& Cooke, 2003; Isaacs, et al., 2003; Jongmans, et al., 1996). En effet, ces habiletés ressortent comme étant particulièrement vulnérables chez les enfants prématurés, spécialement ceux de poids extrêmement faible à la naissance (Dewey, et al., 1999). Ces fonctions sont responsables d'habiletés telles que la coordination papiercrayon, la motricité fine, l'intégration visuo-motrice, la coordination œil-main et la vitesse motrice. Il est donc tout à fait cohérent que les enfants prématurés exhibent des difficultés académiques avec les calculs mathématiques, l'écriture (motricité et orthographe) et la lecture, étant donné l'implication des fonctions visuo-motrices et visuo-spatiales dans ces processus (Aylward, 2002; O'Reilly, et al., 2010). Selon l'étude de Goyen, Lui et Woods (1998), 11 à 20\% des enfants prématurés présenteraient des déficits au niveau des habiletés visuo-perceptuelles et visuo-motrices, alors que cette incidence s'élève à $71 \%$ en ce qui concerne la motricité fine. Ceux-ci peuvent se manifester en dépit d'un QI dans la moyenne (Davis, et al., 2005), ainsi qu'en l'absence d'atteintes neuromotrices (O'Reilly, et al., 2010). Notons, par ailleurs, que plus d'un tiers de ces enfants se voient prescrire des verres correcteurs, en comparaison à seulement $10 \% \mathrm{chez}$ les enfants nés à terme (Saigal, et al., 2001). Enfin, comme pour le QI, on retrouve un gradient de sévérité dans les déficits de ces fonctions, dont la gravité augmente au fur et à mesure que l'âge gestationnel et le poids à la naissance diminuent. (Aylward, 2002).

\section{Attention}

Du côté des fonctions attentionnelles, l'inattention est probablement le déficit cognitif le plus souvent rapporté chez les enfants prématurés (Elgen, et al., 2004; Hack, et al., 2009). L'inattention est observable dès l'âge de trois ans, des chercheurs ayant mis en évidence une attention soutenue plus faible chez les enfants prématurés en comparaison aux enfants nés à terme (Caravale, et al., 2005). La tâche utilisée (i.e. Leiter international performance scale revised) impliquait l'attention en termes de durée et de direction, et s'avère corrélée avec les habiletés de résolution de problème et la 
performance scolaire. Des atteintes de la sphère attentionnelle sont également rapportées à l'âge de six ans (Marlow, et al., 2007). En effet, les enfants prématurés sont significativement plus lents que les enfants nés à terme aux tâches de recherche visuelle, en plus d'éprouver de la difficulté à trouver les cibles et d'exhiber plus de comportements sans lien avec la tâche. Une autre étude a montré la présence de problèmes attentionnels chez des enfants d'âge scolaire pesant $750 \mathrm{~g}$ et moins à la naissance. En effet, ces enfants ont tendance à être plus impulsifs, moins précis et à faire plus d'erreurs dans la détection de cibles comparativement à leur pairs nés à terme (Taylor et al., 1998). Les déficits attentionnels sont aussi rapportés plus tard dans leur scolarité, où les enfants prématurés et de petits poids sont décrits comme étant plus à risque d'avoir un trouble d'attention avec hyperactivité (TDAH) (Aylward, 2002; 2000; Taylor et al., 2000). Taylor et al. (1998) indiquent dans leur étude que les habiletés attentionnelles sont prédictives des capacités d'apprentissage et des comportements à l'âge scolaire; il devient alors primordial d'être sensible à ces aspects auprès des enfants prématurés chez qui la prévalence des troubles attentionnels est plus élevée que chez les enfants nés à terme.

\section{Fonctions exécutives}

Les fonctions exécutives sont aussi rapportées comme étant déficitaires chez les enfants prématurés. Ces fonctions réfèrent à différentes habiletés, soit le contrôle de l'impulsivité, l'autorégulation, l'anticipation, la planification et l'organisation, l'initiation d'une activité, et les stratégies de résolution de problèmes. Chez les enfants prématurés, la totalité de ces fonctions sont atteintes (Anderson, et al., 2004). De façon plus détaillée, plusieurs études montrent que les enfants prématurés et de petits poids âgés de six ans ont des problèmes de planification, d'autorégulation et ont plus de persévération motrice que des enfants du même âge nés à terme (Marlow, et al., 2007). Bayless et Stevenson (2007) en sont arrivés à la même conclusion, mais en précisant que malgré une performance sous la moyenne au Cambridge Neuropsychological Test Assessment Battery (CANTAB) mesurant les fonctions exécutives, on ne pouvait pas parler encore de «déficits » chez les enfants prématurés. Toutefois, la présence concomitante d'un TDAH chez ces derniers augmente la sévérité des troubles des fonctions exécutives comparativement aux enfants prématurés sans TDAH et ce, à toutes les échelles du BRIEF-P (Mahone \& Hoffman, 2007). En plus d'être rapportées à l'âge préscolaire, les perturbations des fonctions exécutives semblent se maintenir à l'âge scolaire, où une étude a montré à ce sujet que les enfants prématurés $(<28$ semaines gestationnelles ou poids $<1000 \mathrm{~g})$ âgés de huit ans ont des difficultés à générer des idées et des stratégies, garder l'information en tête, planifier des actions et organiser l'information et démontrent également des difficultés dans l'initiation des activités (Anderson, et al., 2004; G.H. Taylor, et al., 2000).

\section{Profil comportemental et affectif}

Les troubles comportementaux et les difficultés psychologiques/psychiatriques toucheraient entre 20 et $55 \%$ des enfants prématurés (Anderson et al., 2003; Hack, et al., 1994; Taylor et al., 2000; Valleur, et al., 2004). La littérature rapporte, entre autres, qu'un quart à une moitié des enfants de faible poids et de poids extrêmement faible à la naissance présenteraient des symptômes anxieux et/ou de retrait social (Horwood, et al., 1998), et qu'entre l'âge de 12 et 14 ans, 8 à $14 \%$ d'entre eux répondraient aux critères d'un trouble d'anxiété généralisée (TAG), en comparaison à 1-4\% chez leur pairs (Botting, et al., 1997; Indredavik, et al., 2004; Stephens \& Vohr, 2009). Par ailleurs, des taux plus élevés de comportements internalisés et externalisés sont rapportés chez ces enfants comparativement à ceux nés à terme, en plus des troubles d'hyperactivité et d'inattention qui sont nettement plus fréquents que dans la population générale (i.e. $18 \%$ contre 5\% respectivement) (Baron, et al., 2009; Grunau, et al., 2004; Taylor et al., 2000; Weisglas-Kuperus, et al., 1993). L'ensemble de ces troubles est plus souvent remarqué avec l'entrée à la maternelle, où le comportement de l'enfant est inadapté et rend les périodes d'apprentissage laborieuses. De plus, ces troubles sont souvent combinés avec des difficultés psychologiques telles qu'une plus faible estime de soi, ainsi qu'une confiance plus faible en ce qui à trait à leurs habiletés athlétiques, scolaires, romantiques et professionnelles (Grunau, et al., 2004; Hack, et al., 2009; Taylor et al., 2000; Valleur, et al., 2004). On retrouve également plus de problèmes d'ajustement émotionnel chez les enfants nés prématurément et de petits poids, ainsi qu'une plus grande prévalence de délinquance, de trouble d'opposition et d'agressivité (Anderson \& Doyle, 2008; Hack, et al., 2009; 2000; Taylor et al., 2000). De fait, ces enfants sont donc plus à risque d'être limités dans leurs compétences et leurs habiletés sociales, ainsi que dans leurs comportements adaptatifs (Moster et al., 2008; Taylor et al., 2000). En somme, il semble que les enfants prématurés soient plus à risque d'éprouver des difficultés aux plans comportemental et émotionnel. Toutefois, celles-ci seraient reliées directement ou indirectement au fonctionnement cognitif et aux difficultés d'apprentissages également rapportés chez ces enfants, tel que suggéré par Bayless et al. (2008)

Les troubles d'apprentissages

Compte tenu de la variété de déficits cognitifs et intellectuels rapportés chez les enfants prématurés et de petits poids, il est facile d'en déduire que leurs capacités d'apprentissages et leur performance scolaire s'en trouvent grandement affectées. Les taux élevés de troubles comportementaux et émotionnels contribuent à rendre leur expérience scolaire encore plus ardue et ce, dès leurs premières années. En effet, les enfants prématurés sont trois à cinq fois plus à risque de présenter des difficultés d'apprentissages, les mathématiques et la lecture (compréhension et lecture de mots) apparaissant les plus affectées (de Rodrigues, et al., 2006; O'Callaghan, et al., 1996; Saigal, et al., 2000). Les études qui se sont intéressées aux difficultés scolaires des enfants prématurés ont montré qu'ils performent significativement moins bien que les enfants nés à terme (Anderson et al., 2003; Marlow, et al., 2005; Moster, et al., 2008; Saigal \& Doyle, 2008; Taylor et al., 2000; Taylor, Hack, Klein, et Schatschneider, 1995). Ils exhibent, par exemple, des déficits en ce qui concerne les habiletés visuo-motrices, linguistique (épellation, dénomination, lecture, etc.), ainsi que la résolution de problèmes qui impliquent de multiples fonctions exécutives (i.e. raisonnement, planification). Par ailleurs, il est rapporté 
dans la littérature que les mathématiques, spécialement l'arithmétique et les habiletés numériques, sont particulièrement affectées chez les enfants prématurés, ce qui apparaît cohérent avec la présence d'atteintes des fonctions exécutives et visuo-spatiales qui sont sollicitées lors des calculs mathématiques (Taylor et al., 2000). Dans cette optique, il est donc évident que les enfants prématurés d'âge scolaire nécessitent plus de services d'éducation spécialisée (ex. séances de tutorat dans les cas de troubles d'apprentissage, de langage, etc.) durant leur cheminement; environ $50 \%$ d'entre eux auraient recours à ces ressources, comparativement à $8 \%$ des enfants nés à terme (Taylor et al., 2000). Certains auteurs ont d'ailleurs montré un changement important dans la proportion d'enfants de poids extrêmement faible bénéficiant de services éducationnels spécialisés, passant de temps-partiel à temps plein entre l'âge de 8 ans et l'adolescence où ils semblent moins performants au niveau académique (Saigal, et al., 2000). Ces résultats exposent un fait important, soit qu'un rendement académique satisfaisant à l'âge de 8 ans ne garantit pas un rendement équivalent une fois à l'adolescence.

\section{Discussion}

En résumé, malgré une certaine variabilité d'atteintes possibles chez les enfants prématurés, la littérature appuie l'idée que la prématurité et le petit poids à la naissance constituent deux conditions exerçant une influence néfaste sur le développement global de l'enfant. Malheureusement, une grande proportion des enfants nés prématurément devront faire face à des défis de taille au cours de leur vie, incluant des retards neurodéveloppementaux significatifs, ainsi que des difficultés d'ordre cognitif, comportemental et d'apprentissage. En effet, les études sont nombreuses à cet effet et mettent en évidence des déficits au plan intellectuel, langagier, mnésique, attentionnels, ainsi que des fonctions perceptivo-visuelles, visuo-motrices, visuo-spatiales et exécutives. Il semble également que le fonctionnement émotionnel et comportemental s'en ressent, puisque les enfants prématurés démontrent une plus grande incidence de troubles internalisés et externalisés. Toutefois, une étude prospective à long-terme réalisée par Tideman (2000) montre que chez les enfants modérément prématurés et à faible risque neurologique, les différences au plan cognitif semblent s'atténuer graduellement. Quoi qu'il en soit, la présente revue de littérature souligne l'importance de bien évaluer les enfants prématurés dès leur naissance et d'assurer un suivi à longterme, puisqu'on bon fonctionnement cognitif, émotionnel et comportemental à l'âge préscolaire n'assure pas un bon fonctionnement une fois sur les bancs d'écoles primaire et/ou secondaire.

Avec l'augmentation du nombre de naissances prématurées et du taux de survie, le besoin d'en connaître davantage sur le fonctionnement neurodévelopemental des enfants prématurés à court, moyen et long-termes se fait sentir. Comme ces enfants présentent un risque élevé d'éprouver divers problèmes d'ordre neurologique, développemental, cognitif, émotionnel et comportemental, il apparaît essentiel de leur assurer une surveillance étroite dès la naissance et ce, jusqu'à l'âge adulte, afin de pouvoir fournir les ressources nécessaires aux enfants en cas de besoin. Heureusement, une proportion considérable de ces enfants ne vivra pas de déficits majeurs, ce qui procure de l'espoir à leur famille. Une meilleure compréhension des éléments relatifs à la prématurité, tels que les facteurs périnataux (ex. atteintes cérébrales, neuropathologies), génétiques (ex. genre) et environnementaux, ainsi que leurs impacts sur le développement de l'enfant, s'avère toutefois nécessaire. Ceci permettra, par exemple, d'établir des marqueurs spécifiques permettant d'identifier les déficits de manière précoce auprès des enfants prématurés à risque, et de mettre en place des stratégies préventives et/ou thérapeutiques.

\section{Références}

Allen, M. C. (2002). Preterm outcomes research: a critical component of neonatal intensive care. [Review]. Mental Retardation \& Developmental Disabilities Research Reviews, 8(4), 221-233.

Anderson, \& Doyle, L. W. (2008). Cognitive and educational deficits in children born extremely preterm. [Review]. Seminars in Perinatology, 32(1), 51-58.

Anderson, Doyle, L. W., \& Victorian Infant Collaborative Study, G. (2004). Executive functioning in school-aged children who were born very preterm or with extremely low birth weight in the 1990s. Pediatrics, 114(1), 50-57.

Anderson, P., Doyle, L. W., \& Victorian Infant Collaborative Study, G. (2003). Neurobehavioral outcomes of school-age children born extremely low birth weight or very preterm in the 1990s. JAMA, 289(24), 3264-3272.

Aylward, G. P. (2002). Cognitive and neuropsychological outcomes: More than IQ scores. Mental Retardation and Developmental Disabilities Research Reviews, 8(4), 234-240.

Baron, I. S., Ahronovich, M. D., Erickson, K., Gidley Larson, J. C., \& Litman, F. R. (2009). Age-appropriate early school age neurobehavioral outcomes of extremely preterm birth without severe intraventricular hemorrhage: a single center experience. Early Human Development, 85(3), 191-196.

Bayless, S., Pit-ten Cate, I. M., \& Stevenson, J. (2008). Behaviour difficulties and cognitive function in children born very prematurely. International Journal of Behavioral Development, 32(3), 199-206.

Bayless, S., \& Stevenson, J. (2007). Executive functions in school-age children born very prematurely. Early Human Development, 83(4), 247-254.

Bhutta, A. T., Cleves, M. A., Casey, P. H., Cradock, M. M., \& Anand, K. J. S. (2002). Cognitive and Behavioral Outcomes of School-Aged Children Who Were Born Preterm. JAMA: The Journal of the American Medical Association, 288(6), 728-737.

Botting, N., Powls, A., Cooke, R. W. I., \& Marlow, N. (1997). Attention Deficit Hyperactivity Disorders and Other Psychiatric Outcomes in Very Low Birthweight Children at 12 Years. Journal of Child Psychology and Psychiatry, 38(8), 931-941.

Briscoe, J., Gathercole, S. E., \& Marlow, N. (1998). Short-Term Memory and Language Outcomes After Extreme Prematurity at Birth. J Speech Lang Hear Res, 41(3), 654-666.

Canada, A. d. 1. S. P. d. (2004). À la hauteur : Une mise à jour de la surveillance de la santé des jeunes du Canada Retrieved 5 septembre, 2010, from http://www.phac-aspc.gc.ca/publicat/meashaut/mu d-fra.php

Caravale, B., Tozzi, C., Albino, G., \& Vicari, S. (2005). Cognitive development in low risk preterm infants at 3-4 years of life. Arch Dis Child Fetal Neonatal 90(6), F474-F479.

Davis, D. W., Burns, B. M., Wilkerson, S. A., \& Steichen, J. J. (2005). Visual Perceptual Skills in Children Born With Very Low Birth Weights. Journal of Pediatric Health Care, 19(6), 363-368.

de Rodrigues, M. C. C., Mello, R. R., \& Fonseca, S. C. (2006). Learning diffuclties in schoolchildren born with very low birth weight. Jornal de Pediatria, 82(1), 6-14.

Dewey, D. G., Crawford, S. G., Creighton, D. E., \& Sauve, R. S. (1999). Long-term neuropsychological outcomes in very low birth weight children free of sensorineural impairments. Journal of Clinical \& Experimental Neuropsychology: Official Journal of the International Neuropsychological Society, 21(6), 851-865.

Doyle, L. W., \& the Victorian Infant Collaborative Study Group. (2004). Evaluation of Neonatal Intensive Care for Extremely Low Birth Weight Infants in Victoria Over Two Decades: I. Effectiveness. Pediatrics, 113(3), 505-509. 
du Plessis, A. J., \& Volpe, J. J. (2002). Perinatal brain injury in the preterm and term newborn. [Review]. Current Opinion in Neurology, 15(2), 151-157.

Elgen, I., Lundervold, A. J., \& Sommerfelt, K. (2004). Aspects of inattention in low birth weight children. Pediatric Neurology, 30(2), 92-98.

Foulder-Hughes, L. A., \& Cooke, R. W. I. (2003). Motor, cognitive, and behavioural disorders in children born very preterm. Developmental Medicine \& Child Neurology, 45(2), 97-103.

Goyen, T.-A., Lui, K., \& Woods, R. (1998). Visual-motor, visual-perceptual, and fine motor outcomes in very-low-birthweight children at 5 years. Developmental Medicine \& Child Neurology, 40(2), 76-81.

Grunau, R. E., Whitfield, M. F., \& Fay, T. B. (2004). Psychosocial and Academic Characteristics of Extremely Low Birth Weight $(\leq 800$ g) Adolescents Who Are Free of Major Impairment Compared With Term-Born Control Subjects. Pediatrics, 114(6), e725-e732.

Guarini, A., Sansavini, A., Fabbri, C., Alessandroni, R., Faldella, G., \& Karmiloff-Smith, A. (2009). Reconsidering the impact of preterm birth on language outcome. Early Human Development, 85(10), 639-645.

Hack, M., \& Taylor, H. G. (2000). Perinatal Brain Injury in Preterm Infants and Later Neurobehavioral Function. JAMA: The Journal of the American Medical Association, 284(15), 1973-1974.

Hack, M., Taylor, H. G., Klein, N., Eiben, R., Schatschneider, C., \& MercuriMinich, N. (1994). School-age outcomes in children with birth weights under $750 \mathrm{~g}$. New England Journal of Medicine, 331(12), 753-759

Hack, M., Taylor, H. G., Schluchter, M., Andreias, L., Drotar, D., \& Klein, N. (2009). Behavioral outcomes of extremely low birth weight children at age 8 years. Journal of Developmental \& Behavioral Pediatrics, 30(2), 122-130.

Horwood, L., Mogridge, N., \& Darlow, B. (1998). Cognitive, educational, and behavioural outcomes at 7 to 8 years in a national very low birthweight cohort. 79(1), F12-F20.

Indredavik, M., Vik, T., Heyerdahl, S., Kulseng, S., Fayers, P., \& Brubakk, A. (2004). Psychiatric symptoms and disorders in adolescents with low birth weight. Arch Dis Child Fetal Neonatal Ed, 89(5), F445450 .

Isaacs, E. B., Edmonds, C. J., Chong, W. K., Lucas, A., \& Gadian, D. G. (2003). Cortical anomalies associated with visuospatial processing deficits. Annals of Neurology, 53(6), 768-773.

Jacobs, S. E., O'Brien, K., Inwood, S., Kelly, E. N., \& Whyte, H. E. (2000). Outcome of infants 23-26 weeks' gestation pre and post surfactant. Acta Paediatrica, 89(8), 959-965.

Jansson-Verkasalo, E., Korpilahti, P., Jantti, V., Valkama, M., Vainionpaa, L., Alku, P., et al. (2004). Neurophysiologic correlates of deficient phonological representations and object naming in prematurely born children. [Clinical Trial]. Clinical Neurophysiology, 115(1), 179-187.

Johnson, S. (2007). Cognitive and behavioural outcomes following very preterm birth. Seminars in Fetal and Neonatal Medicine, 12(5), 363-373.

Jongmans, M., Mercuri, E., Henderson, S., de Vries, L., Sonksen, P., \& Dubowitz, L. (1996). Visual function of prematurely born children with and without perceptual-motor difficulties. Early Human Development, 45(1-2), 73-82.

Le Normand, M.-T., \& Cohen, H. (1999). The delayed emergence of lexical morphology in preterm children: the case of verbs. Journal of Neurolinguistics, 12(3-4), 235-246.

Mahone, E. M., \& Hoffman, J. (2007). Behavior ratings of executive function among preschoolers with ADHD. Clinical Neuropsychologist, 21(4), 569-586.

Marlow, N., Hennessy, E. M., Bracewell, M. A., Wolke, D., \& Group, E. P. S. (2007). Motor and executive function at 6 years of age after extremely preterm birth. Pediatrics, 120(4), 793-804

Marlow, N., Wolke, D., Bracewell, M. A., Samara, M., \& Group, E. S. (2005). Neurologic and developmental disability at six years of age after extremely preterm birth. New England Journal of Medicine, 352(1), 9-19.

Méio, M. D., Lopes, C. S., Morsch, D. S., Monteiro, A. P. G., Rocha, S. B., Borges, R. A., et al. (2004). Pre-school cognitive development of very low birth weight preterm children. Jornal de Pediatria, 80(6), 495-502.

Ment, L. R., Vohr, B., Allan, W., Westerveld, M., Katz, K. H., Schneider, K. C., et al. (1999). The etiology and outcome of cerebral ventriculomegaly at term in very low birth weight preterm infants. Pediatrics, 104(2 Pt 1), 243-248.
Moster, D., Lie, R. T., \& Markestad, T. (2008). Long-term medical and social consequences of preterm birth. New England Journal of Medicine, 359(3), 262-273.

Nolin, P., \& Laurent, J.-P. (2004). Neuropsychologie cognition et développement de l'enfant. Sainte-Foy Que.: Presses de l'Université du Québec.

O'Callaghan, M. J., Burns, Y. R., Gray, P. H., Harvey, J. M., Mohay, H., Rogers, Y. M., et al. (1996). School performance of ELBW children: a controlled study Developmental Medicine \& Child Neurology, 38(10), 917-926.

O'Reilly, M., Vollmer, B., Vargha-Khadem, F., Neville, B., Connelly, A., Wyatt, J., et al. (2010). Ophthalmological, cognitive, electrophysiological and MRI assessment of visual processing in preterm children without major neuromotor impairment. Developmental Science, 13(5), 692-705.

Raz, S., Debastos, A. K., Newman, J. B., \& Batton, D. (2009). Extreme prematurity and neuropsychological outcome in the preschool years. of the international neuropsychological society, 16, 169179.

Roberts, G., Anderson, P. J., \& Doyle, L. W. (2009). Neurosensory Disabilities at School Age in Geographic Cohorts of Extremely Low Birth Weight Children Born Between the 1970s and the 1990s. The Journal of Pediatrics, 154(6), 829-834.e821.

Saigal, S., \& Doyle, L. W. (2008). An overview of mortality and sequelae of preterm birth from infancy to adulthood. The Lancet, 371(9608), 261-269.

Saigal, S., Hoult, L. A., Streiner, D. L., Stoskopf, B. L., \& Rosenbaum, P. L. (2000). School Difficulties at Adolescence in a Regional Cohort of Children Who Were Extremely Low Birth Weight. Pediatrics, 105(2), 325-331.

Saigal, S., Stoskopf, B. L., Streiner, D. L., \& Burrows, E. (2001). Physical Growth and Current Health Status of Infants Who Were of Extremely Low Birth Weight and Controls at Adolescence. Pediatrics, 108(2), 407-415

Saigal, S., Szatmari, P., Rosenbaum, P., Campbell, D., \& King, S. (1990). Intellectual and functional status at school entry of children who weighed 1000 grams or less at birth: A regional perspective of births in the 1980s. The Journal of Pediatrics, 116(3), 409-416.

Santé, O. M. d. 1. (2010). Incidence mondiale de la naissance avant terme : revue systématique de la mortalité et de la morbidité maternelle Retrieved 5 septembre 2010, 2010

Scherjon, S., Briet, J., Oosting, H., \& Kok, J. (2000). The discrepancy between maturation of visual-evoked potentials and cognitive outcome at five years in very preterm infants with and without hemodynamic signs of fetal brain-sparing. Pediatrics, 105(2), 385391.

Scientist, N., \& Reuters. (2007). World's most premature baby set to leave hospital Retrieved 5 septembre 2010, from http://www.newscientist.com/article/dn11222-worlds-mostpremature-baby-set-to-leave-hospital.html

Stephens, B. E., \& Vohr, B. R. (2009). Neurodevelopmental Outcome of the Premature Infant. Pediatric Clinics of North America, 56(3), 631646.

Szatmari, P., Saigal, S., Rosenbaum, P., Campbell, D., \& et al. (1990). Psychiatric disorders at five years among children with birthweights <1000g: A regional perspective. Developmental Medicine \& Child Neurology, 32(11), 954-962.

Taylor, Minich, N., Bangert, B., Filipek, P. A., \& Hack, M. (2004). Longterm neuropsychological outcomes of very low birth weight: associations with early risks for periventricular brain insults. Journal of the International Neuropsychological Society, 10(7), 987-1004

Taylor, G. H., Hack, M., \& Klein, N. K. (1998). Attention Deficits in Children with $<750$ gm Birth Weight. Child Neuropsychology, 4(1), 21 - 34.

Taylor, G. H., Klein, N., \& Hack, M. (2000). School-age consequences of birth weight less than $750 \mathrm{~g}$ : a review and update. Developmental Neuropsychology, 17(3), 289-321.

Taylor, G. H., Klein, N., Minich, N. M., \& Hack, M. (2000). Middle-SchoolAge Outcomes in Children with Very Low Birthweight. Child Development, 71(6), 1495-1511.

Taylor, G. H., Klein, N., Minich, N. M., \& Hack, M. (2000). Verbal Memory Deficits in Children with Less than $750 \mathrm{~g}$ Birth Weight. Child Neuropsychology, 6(1), 49 - 63.

Taylor, G. H., Minich, N., Bangert, B., Filipek, P., \& Hack, M. (2004). Longterm neuropsychological outcomes of very low birth weight: Associations with early risks for periventricular brain insults. 
Journal of the International Neuropsychological Society, 10(7), 987-1004.

Taylor, H. G., Hack, M., Klein, N., \& Schatschneider, C. (1995). Achievement in Children with Birth Weights Less Than 750 Grams with Normal Cognitive Abilities: Evidence for Specific Learning Disabilities. Journal of Pediatric Psychology, 20(6), 703-719.

Tideman, E. (2000). Longitudinal follow-up of children born preterm: cognitive development at age 19. Early Human Development, 58, 81-90.

Valleur, D., Magny, J.-F., Rigourd, V., \& Kieffer, F. (2004). Le pronostic neurologique à moyen et long terme des prématurés d'âge gestionnel inférieur à 28 semaines d'aménorrhée. European Journal of Obstetrics \& Gynecology and Reproductive Biology, 33, 1S72-71S78.

Vicari, S., Caravale, B., Carlesimo, G. A., Casadei, A. M., \& Allemand, F. (2004). Spatial Working Memory Deficits in Children at Ages 3-4 Who Were Low Birth Weight, Preterm Infants. Neuropsychology, 18(4), 673-678.

Volpe, J. J. (2001). Perinatal brain injury: from pathogenesis to neuroprotection. Mental Retardation \& Developmental Disabilities Research Reviews, 7(1), 56-64.

Weisglas-Kuperus, N., Koot, H. M., Baerts, W., Fetter, W. P. F., \& Sauer, P. J. J. (1993). Behaviour problems of very lowbirthweight children. Developmental Medicine \& Child Neurology, 35(5), 406-416.

Wolke, D., Samara, M., Bracewell, M., Marlow, N., \& Group, E. P. S. (2008). Specific language difficulties and school achievement in children born at 25 weeks of gestation or less. Journal of Pediatrics, 152(2), 256-262. 